(Supporting Information)

\title{
Cooperative Vinculin Binding to Talin Mapped by Time Resolved Super Resolution Microscopy
}

Xian Hu1, Chaoran Jing2, Xiaochun Xu1, Naotaka Nakazawa1, Virginia W. Cornish2, Felix M. Margadant1 and Michael P. Sheetz1,3,*

[*] Prof. M.P.Sheetz

1Mechanobiology Institute, National University of Singapore, Singapore 117411, Singapore

2Department of Chemistry, Columbia University, New York, New York 10027, USA

3Department of Biological Sciences, Columbia University, New York, New York 10027, USA

*Correspondence should be addressed to M.P.S(email: ms2001@columbia.edu) 


\section{SUPPLEMENTARY MOVIE LEGENDs}

Supplementary Movie 1 Talin Single Molecule Trace: Processed movie of individual single talin pair from 15 seconds of actual data (GFP-talin-mCherry( Green:GFP, Red:mCherry)).

Supplementary Movie 2 Real time three channel process chain. Talin Single Molecule Trace vs. Vinculin count events: A. Maximum Intensity Projection (MIP) overview of the two channels of GFP-talin-mCherry (Green:GFP, Red:mCherry). B. MIP of Vinculin Atto655. C. Enlarged movie of the single molecule Bayesian of the GFP-talin-mCherry channels in the marked Region of Interest (ROI). D. Enlarged movie of the Vinculin Atto655 channel in the same ROI. E. Identified single molecule pairs from panel C. F Identified molecular count from panel D. All scale bars are $1 \mu \mathrm{m}$, the observation lasts 17 seconds.

Supplementary Movie 3 Talin Single Molecule Trace and Vinculin molecular count: Processed 19 second movie of the interaction of an individual single talin pair and Vinculins from actual data. (GFP-talin-mCherry (Green:GFP, Red:mCherry), Vinculin Atto655(Purple)).

\section{MATERIAL AND METHODS}

\section{Cell Culture, Plasmid, Transfection and Dye Characterization}

Talin depleted cells ${ }^{1}$ were cultured in DMEM (Gilbco,Life Technologies), 10\% FBS(Hy-Clone, GE Healthcare) in tissue culture incubators that were maintained at $5 \% \mathrm{CO} 2$ and $37^{\circ} \mathrm{C}$. The GFP-talin-mCherry plasmid has been previously described ${ }^{2}$ (all talin construct used in this manuscript is talin1). Wild type vinculin (WT_vinculin), vinculin A50I, vinculin head (obtained from Dr. Susan Craig's Laboratory) and myosin light chain (MLC) were genetically engineered with DHFR:L28C in-house. 
Construction of DHFR:L28C-vinculin vector: The gene encoding DHFR:L28C was amplified using PCR from the previously published $\mathrm{p} 2250^{3}$ with primers DHFR-AgeI: 5'- CCA CCG GTA CCA TGA TCA GTC TGA TTG CGG CGT TAG -3' (AgeI, coding strand) and DHFR-SacI: 5'- CCG AGC TCG GCC AGA ACC ACC AGA CCG CCG CTC CAG AAT CTC AAA GC -3' (NotI, noncoding strand). The fragment was then inserted between the AgeI and SacI sites of vector encoding EGFP-vinculin replacing the EGFP gene. The synthesis of A-TMP-Atto655 was described in ${ }^{4}$, but the conventional incubation-staining method doesn't work very well for fibroblasts. Electroporation (1250v, 20ns, 1 pulse)(Neon, Life Technologies) was deployed to introduce both plasmids-of-interest and A-TMP-Atto655 dye molecules into the cells, 24 hours before imaging. Concentration of constructs and the dye used for electroporation per $10 \mu \mathrm{l}$ of cell suspension: GFP-talin-mCherry (100ng/ $\mu \mathrm{l})$, DHFR constructs (1000ng/ $\mu \mathrm{l})$, A-TMP-Atto655(20 $\mu \mathrm{M})$. This optimization of the staining protocol combines staining and transfection into one single step 24 hours before imaging, which results in a much simpler experiment procedure as well as allowing the cells to have sufficient rest after electroporation. The MLC-DHFR: L28C ATMP-Atto655 marks the stress fiber in RPTP alpha ${ }^{+/+}$MEF cells ${ }^{5}$ (Figure 2A), indicating that the staining system doesn't affect the natural localization of the protein of interest, and the dye highlights the protein of interest within the first 48 hours after transfection both in live cells and fixed cells.

The A-TMP-Atto655 has a favorable low blinking frequency for the purpose of counting. In a sparse sample, well-isolated signals were chosen by the criterion that they go dark within two frames - this ensures that the signal originates from a single emitter. Fixation and fiducials are important to assure the sample doesn't drift and we do not misidentify different molecules for the original one.

Unlike Atto655 in oxygen rich solutions, our samples in adhesions featured blinking intervals (dark times) of mostly 5 to 20 seconds (with the bulk around 8-12s), which makes them longlived enough for counting 'on' blimps upon recruitment. The method fails occasionally: we observed echoes in a single stretch cycle where bursts of near identical relative positions and numbers re-occur after some 8 seconds. We discarded such events when the software detected them. 
Cells were reseeded 2 hours before imaging from plastic cell culture dishes to fibronectin (EMD Millipore) coated round cover glasses and fitted into live cell-imaging chambers (Bioptechs, 10mm Interchangeable Coverslip Dish) with normal cell culture media as described above. Fiducials (Sphero multiple-fluorophore, Spherotch Inc.) were added into the chamber 10 minutes before imaging. 


\section{Western Blotting}

A Western Blot was performed to determine the ratio of vinculin-DHFR versus endogenous vinculin in this experiment. The ratio of endogenous vinculin versus vinculin-DHFR is $6.85+/-$ 3.07 .

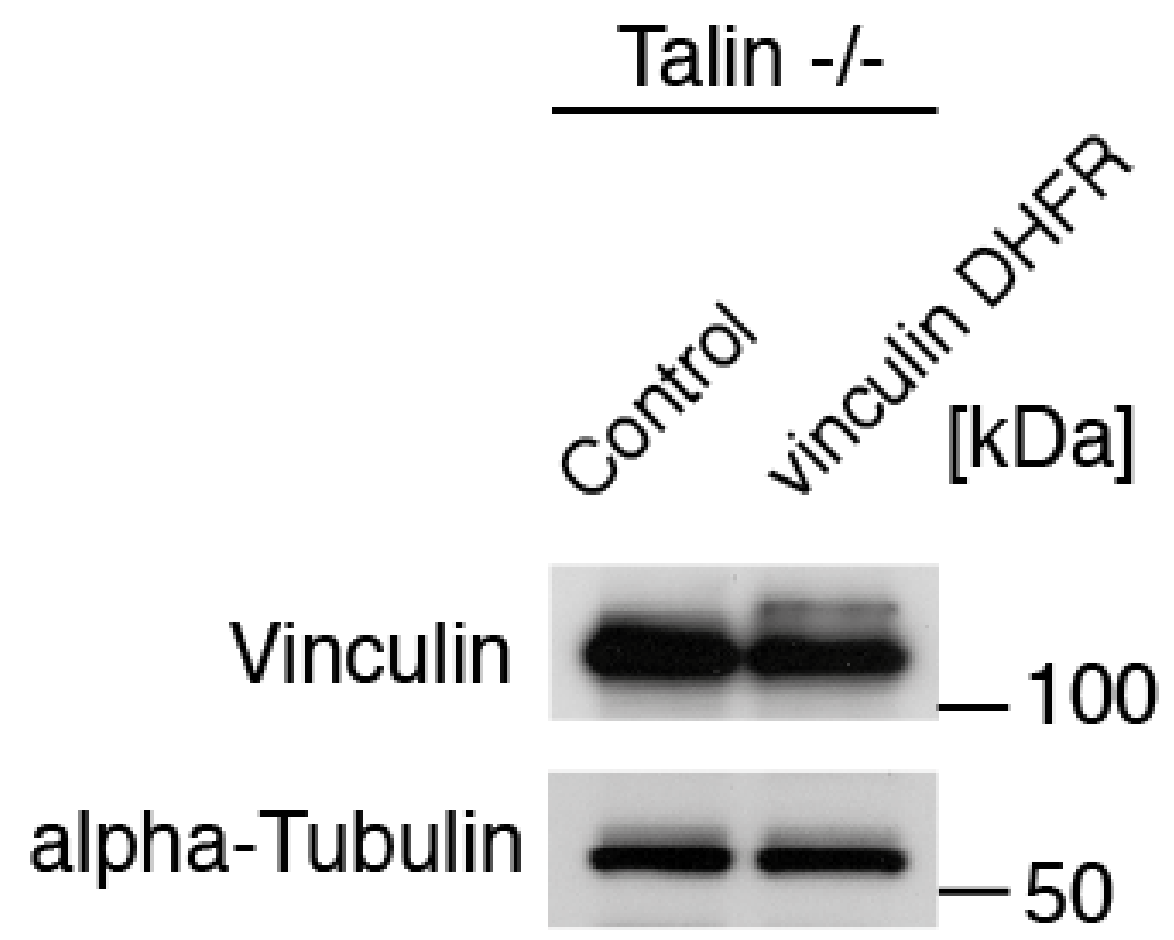

Supplementary Figure 1 Western blot of expression level of DHFR-vinculin (145kDa) versus endogenous vinculin(120kDa) 


\section{Microscopy}

All quantitative imaging was performed in TIRF microscopy. The relevant observations occur well within the evanescent field of the internal reflection and since our localization approach suffers from and is limited by background contamination, which is unavoidable in intact, spreading fibroblast, this conservative, low efficiency mode is justified.

Atto655 labeled vinculin, once recruited to talin and activated (dye activation), was localized by its bright flare under intense $647 \mathrm{~nm}$ illumination. Within $30-60 \mathrm{~ms}$, the Atto655 cycled into the triplet state allowing for a decent time resolution $(<30 \mathrm{~ms})$ and a simple and accurate localization described below.

The Atto655 channel was imaged at $33 \mathrm{~ms}$ per frame streaming mode, i.e. with no temporal gap between consecutive frames, with the exception when the GFP and mCherry channels were imaged once a second with a short exposure time onto the same EMCCD via a single multiband filter set. Channel selection was achieved by AOTF switching of the laser line and galvo adjustment of the TIRF angle and direction. That is twice a second the 647nm pump was interrupted and the GFP and mCherry labels respectively were read out. As the labels needed to be tracked for 15-60 exposures, low doses and short exposures of 5-20ms with $491 \mathrm{~nm}$ and $561 \mathrm{~nm}$ light respectively were administered. Thereby, the talin stretch and spatial orientation was assessed once a second.

Calibration experiments were performed on a Nikon NSTORM Ti Sapphire scope with Nikon CFi TIRF lenses with 1.49 numerical aperture and 60x and 100x magnification respectively. The system was equipped with a single Andor 897 back-illuminated EMCCD camera with a $10 \mathrm{MHz}$ readout cap and 16 micrometer pixels paired with a $1.5 \mathrm{x}$ tube magnifier that produced a back projected pixel size of $107 \mathrm{~nm}$ and an averaged frame rate not exceeding 37fps at full chip exposure. Thanks to the built in 500 microseconds frame transfer, two short exposures can be acquired in just under $20 \mathrm{~ms}$ - before the data has to be read out sequentially - but the software control limited the timing accuracy and repeatability. At $20 \mathrm{~ms}$ or longer exposures, however, the timing was dependable and steady. The TIRF angle adjustment was manual, so sequences had to be recorded at a suitable intermediate setting so that penetration depths could not be 
quantitatively repeated between experiments. TIRF light could only be fed from one side of the lens and fringe suppression was achieved by a collimator and circular polarization which itself increased the uncertainty of the penetration depth.

The $647 \mathrm{~nm}$ line was a $150 \mathrm{~mW}$ diode and the $488 \mathrm{~nm}$ and $561 \mathrm{~nm}$ sources were provided from $100 \mathrm{~mW}$ DPSS lasers and as multi-Argon lines, rated at $28 \mathrm{~mW}$ and $7 \mathrm{~mW}$ respectively.

In order to combine the large difference in power needed between the GFP-mCherry length measurements and the vinculin recruitment detection as well as to accommodate higher timing accuracy at short exposures with better cross-talk removal, for the actual real-time recordings, we used dual two-axis galvo beam steering controllers from Roeper Scientific on an Olympus X81 body. The steering allowed traversal of the backfocal plane in 3ms, and a peripheral ring generating a TIRF beam from all directions at the specimen - in 6.5ms. Quarter circles - the minimum travel to suppress fringes entirely - and changes between channels were done in $2 \mathrm{~ms}$.

The laser sources provide diode lines for $405 \mathrm{~nm}$ and $647 \mathrm{~nm}$ at $100 \mathrm{~mW}$ and DPPS for $491 \mathrm{~nm}$ and 561 at $200 \mathrm{~mW}$. The quad dichroic and emitter have center transmission lines of $416 \mathrm{~nm}, 500 \mathrm{~nm}$, $582 \mathrm{~nm}$, and $657 \mathrm{~nm}$. The two cameras are split by a single dichroic into the $523 \mathrm{~nm}$ or $647 \mathrm{~nm}$ signal. The system including the stage was immersed in a customized blackened weather chamber with high walls to minimize air flow across the stage.

The system was upgraded to two Photometrics Evolve Delta cameras separated by a slim dichroic mirror on a Photometrics DC2 dual camera mount. Camera adjustments proved too unstable and crude to provide localization microscopy and special calibration assays were used. The advantage of a two camera system was that the switching to record the green and red tracking images could be performed in minimum time and that one camera would handle the bright dSTORM channel and the other the dim tracking images. But when exposure times of $30 \mathrm{~ms}$ of greater were used for red and green acquisitions, a single camera could be cleared easily and the superior calibration of a single camera proved to be favorable. We used both techniques as dictated by the speed-of-acquisition criterion. Fast exposures were recorded on two cameras.

All exposure times were chosen to be integer multiples of the beam rotation time, i.e. multiples of $2 \mathrm{~ms}$ and $6.5 \mathrm{~ms}$ respectively, deviating from the timing led to subtle apparent shaking of the entire scene and brightness fluctuations. Short and accurate exposure times proved to be 
undoable with our software tools (Roeper AOTF shutter, attenuator, and wavelength selection) until a hardware delay line was added that enforced beam steering speed and shutter times. The ideal exposure time of $33 \mathrm{~ms}$ was shortened to $5 \times 6.5 \mathrm{~ms}=32.5 \mathrm{~ms}$ with the frame transfer only blanking the system for some $1.5 \%$ of the time. The shorter streaks of multiples of $2 \mathrm{~ms}$ delivered a clean TIRF image but rendered the Metamorph control software unstable, they were hence not used for the later datasets.

The galvo drives took hours to reach a thermal steady state before they stopped drifting and randomizing the imaging and were therefore kept on. 


\section{Image Processing}

Calibration and reference data were obtained during the experimental period. The systems' PSFs for different wavelengths were acquired in between experiments. Chromatic matching was performed via sub-diffraction beads (Sphero multiple-fluorophore, Spherotch Inc.) at high density between experiments and as isolated fiducials within experiments. For the latter, the beads were sparse and settled on the cover slip to provide focus and xy-drift compensation to tell substrate and actin flow movement apart. All recordings were made in Nyquist over-sampled images. Photon counting was achieved by analyzing the noise pattern on the Andor897 camera or by using the photo-conversion calibration on the Photometrics Evolve cameras. The conversion multiplication drifted strongly over time and needed to be reestablished regularly.

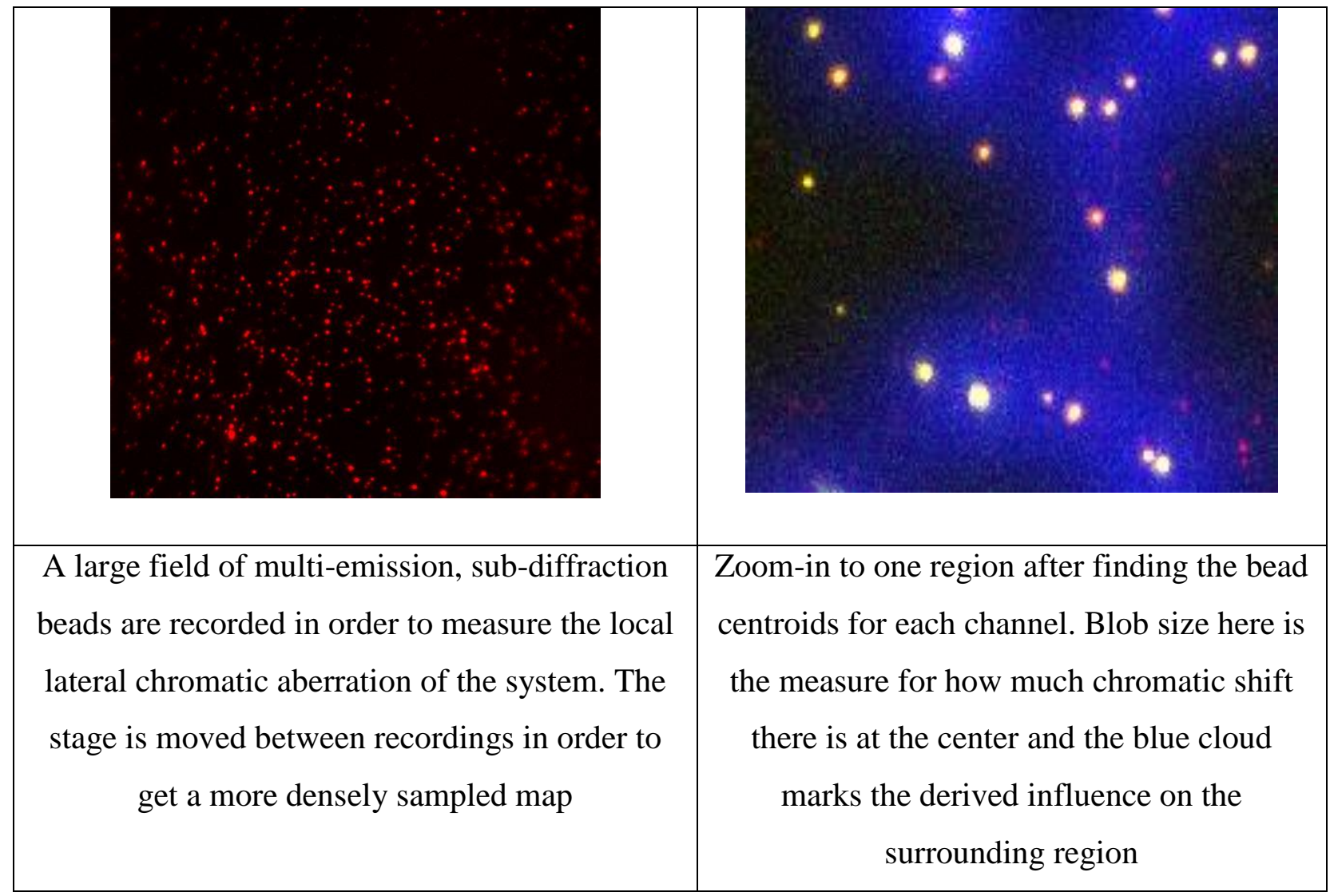

Supplementary Figure 2 Measurement and calculation of the chromatic aberration map needed to realign all three spectral channels to superresolution accuracy

Spectral separation was used to distinguish the GFP and mCherry labels. All localizations were coarsely determined with an autocorrelation filter that delivers a fitted centroid, followed by a Maximum Likelihood (ML) matching of the measured PSF of the corresponding channel (see 
illustration micrograph below) to the observed signal. We used both measured and calculated PSFs (high NA vectorial) for the analysis. Measured PSFs are obtained from sparse images whose signals are confirmed to be single fluorophores by the observation of single step bleaching events (i.e. the signal had full strength in frame $n$ and was entirely gone in frame $n+2$, assuring that the molecule became nonfluorescent during exposure $n+1$ ). Computed PSFs were obtained as described in the "Handbook of Biological Confocal Microscopy" 2006 (ISBN 9780387259215), chapter 11 "Measuring the Real Point Spread Function of High Numerical Aperture Microscope Objective Lenses” by Rimas Juskaitis.

We used a two-stage algorithm to estimate the separation of the two N-terminal GFPs. It first derived an estimate for the relative coordinates of the individual GFP emitters from the eccentricity of the observed dual PSF and then iteratively fitted a maximum likelihood model of two isolated PSFs to it.

The iterative algorithm was not the subject of this paper, the quantification of the accuracy though was of essence here. We calibrated and tested the approach by means of a single emitter that was translated via a piezo nanostepping stage by a pre-set displacement. The Physik Intrumente P-733 capacitive controlled piezo stage featured 30x30x10 micron travel and a repeat accuracy of $1 \mathrm{~nm}$ or better
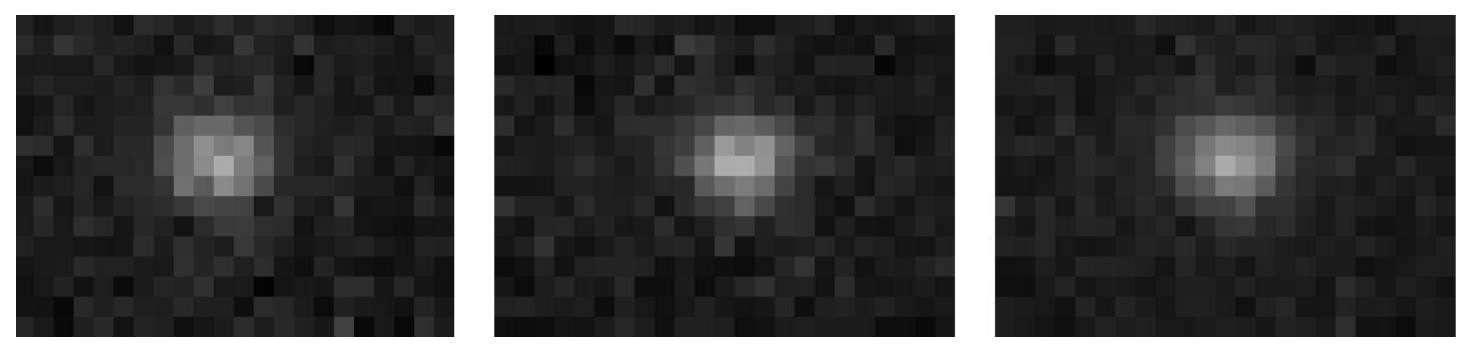

Supplementary Figure 3 First exposure, $2^{\text {nd }}$ exposure $+x 100 \mathrm{~nm}$ and sum of both images

In this case two images with the same exposure properties (power, wavelength, and time) were recorded with a defined displacement in between. The images were then summed and analyzed by the two-stage algorithm. This was for illustration purposes, to obtain a more realistic noise pattern, the later recordings were performed in a single exposure. The movement time of the 
stage was below $2 \mathrm{~ms}$ giving a finely calibrated test image. The inertia and shaking of piezo stages were usually significant but at $100 \mathrm{~nm}$ or less travel it proved to be not noticeable.

The fitting algorithm was computationally expensive and has no natural convergence - it kept oscillating around all possible values and often the possible configurations had to be found by exhaustive search which routinely led to the comparison of 1000 to 40000 constellations per time point. Fortunately, not that many clean GFP dimer signals were found per experiment and processing of 512 square frames was be performed in $10 \%$ of real time on an Intel i7 $4700 \mathrm{~K}$ CPU with 32GB main memory and an Nvidia GeForce 980 graphics engine.

The performance was inversely proportional to the actual separation of the two emitters. At 500 photons collected, the accuracy was less than $25 \mathrm{~nm}-35 \mathrm{~nm}$ for a separation of $70 \mathrm{~nm}$ of more. But grew to nearly twice that for separations of 30nm or less. Noise and defocus also inevitably led to the algorithm overestimating the actual separation - the error was highly asymmetrical in nature, it dominantly erred in favor of longer separations.

The errors obtained from very small beads, high yield (1K photons), and averaged 500 times in order to obtain a smooth curve, are given here and also illustrate how the method benefits from higher photon output.

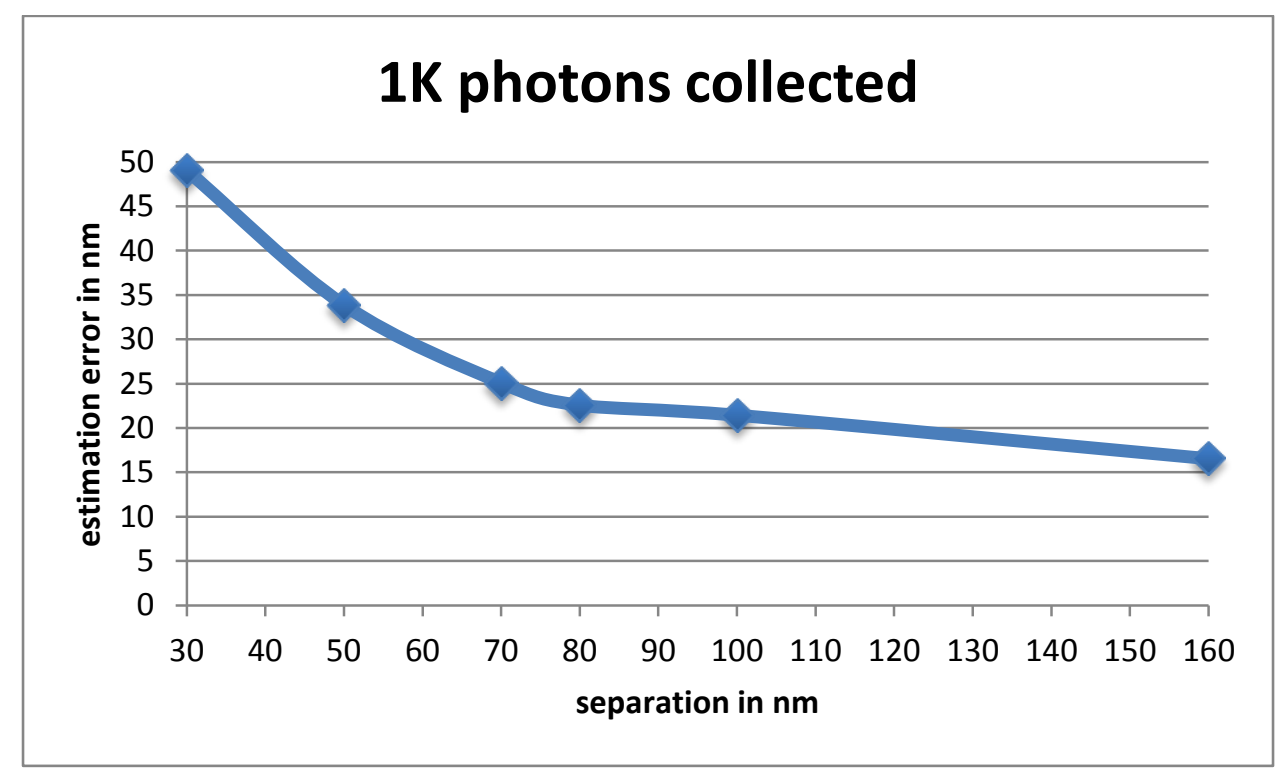

Supplementary Figure 4 Resolving power of the dimer localization algorithm vs. the actual separation of the dimers 
The third channel, the blinking Atto655, was tracked with our standard dSTORM software that also employed autocorrelation, ML matching, and then temporal isolation and re-occurrence detection of blinking signals. The tracking feature had little relevance for this work other than counting fast-returning dye molecules so that they were only counted once per measurement. The methods while similar in nature yielded localizations of different accuracy. The vinculin signal was localized to $8-25 \mathrm{~nm}$, the N-to-C stretch to about $20-24 \mathrm{~nm}$ at high power, and the N-to-N separation followed the complex dependency discussed above. 


\section{Statistics and Reliability of the Data Processing}

The measurement of specificity: the statements derived in this work rely on the tagged vinculin not efficiently binding to a component other than talin. For this test, we use the early frames of a sample that has not been exposed and bleach before. The chances of all four fluorophores of an intact talin dimer not being fluorescent should then range in the low $1 / 10^{\text {th }}$ of a percent. Vinculin blinking events are then correlated to their proximity of a talin signal. If the event occurred within one PSF radius of the talin, it is counted as an on-talin event otherwise as a nonspecific count. The test is insensitive to the amount of non-labeled vinculin but it does overestimate specificity as the PSF radius is somewhat larger than the length of the talin molecule. It should deliver a more realistic result on and around a talin-rich focal adhesion as the test then reduces to a simple in-out test. For the intact and well-defined adhesions of the reference full length vinculin/talin pair, this specificity ratio scored in the 100 to 115 range.

For the A50I mutant talin, the ratio drops significantly and it depends on the structure of the adhesions:

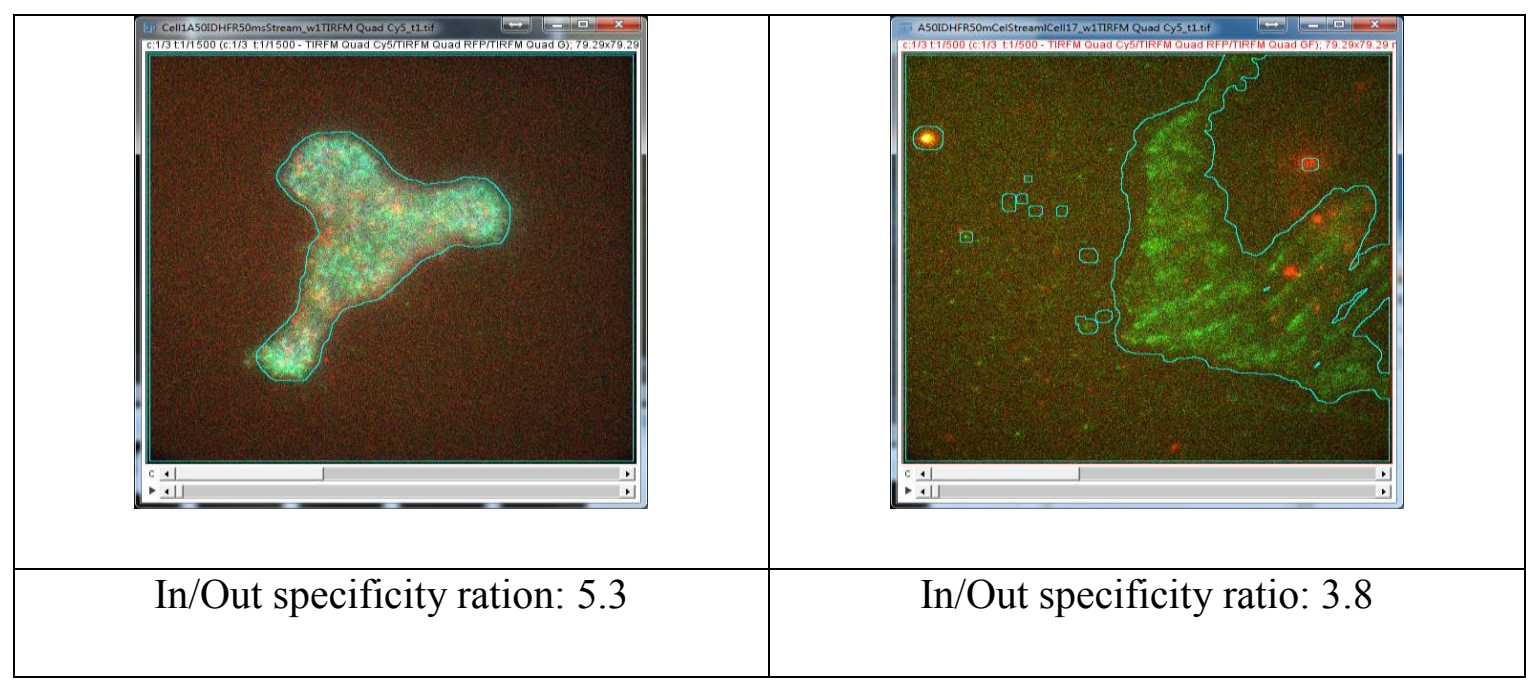

Supplementary Figure 5 Specificity of A50I vinculin mutant binding to GFP-talin-mCherry for different adhesion types

Without bleaching of the GFP-mCherry tags on the talin, these ratios should behave inversely.

For the vinculin head construct, this dependency varies a lot more: 


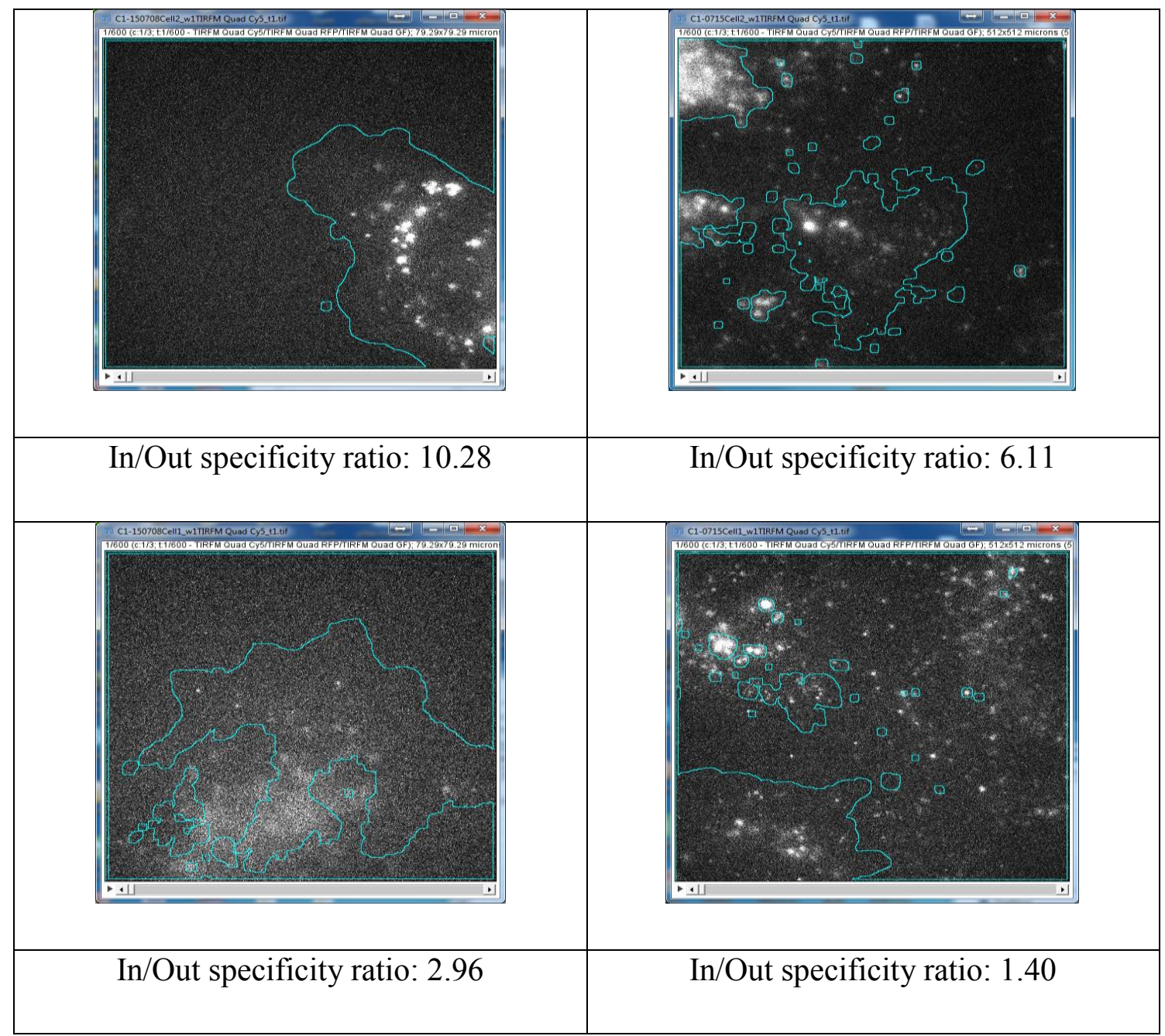

Supplementary Figure 6 Specificity of vinculin head binding to GFP-talin-mCherry for different adhesion types

In the fragmented adhesions, nonspecific binding clearly does occur.

Measurement of this preferred terminal for recruitment: we examined whether the vinculin binding sites were near the $\mathrm{C}$ terminus or the $\mathrm{N}$-terminus as a function of the stretch length. To illustrate the landscape, we plotted the histograms how many recruitments in our observations happen closer to $\mathrm{C}$ verse those that happen closer to $\mathrm{N}$, which were plotted against the average stretch state of the talin at the time of observation in 20nm buckets with bucket $\mathrm{n}$ being [ $n-$ 1) $\cdot 20 \mathrm{~nm} . . n \cdot 20 \mathrm{~nm}$ [ in the $\mathrm{x}$-axis whereas the $\mathrm{y}$-axis is the total count of vinculin registration that hit closer to $\mathrm{C}$ (red) and closer to $\mathrm{N}$ (green). 


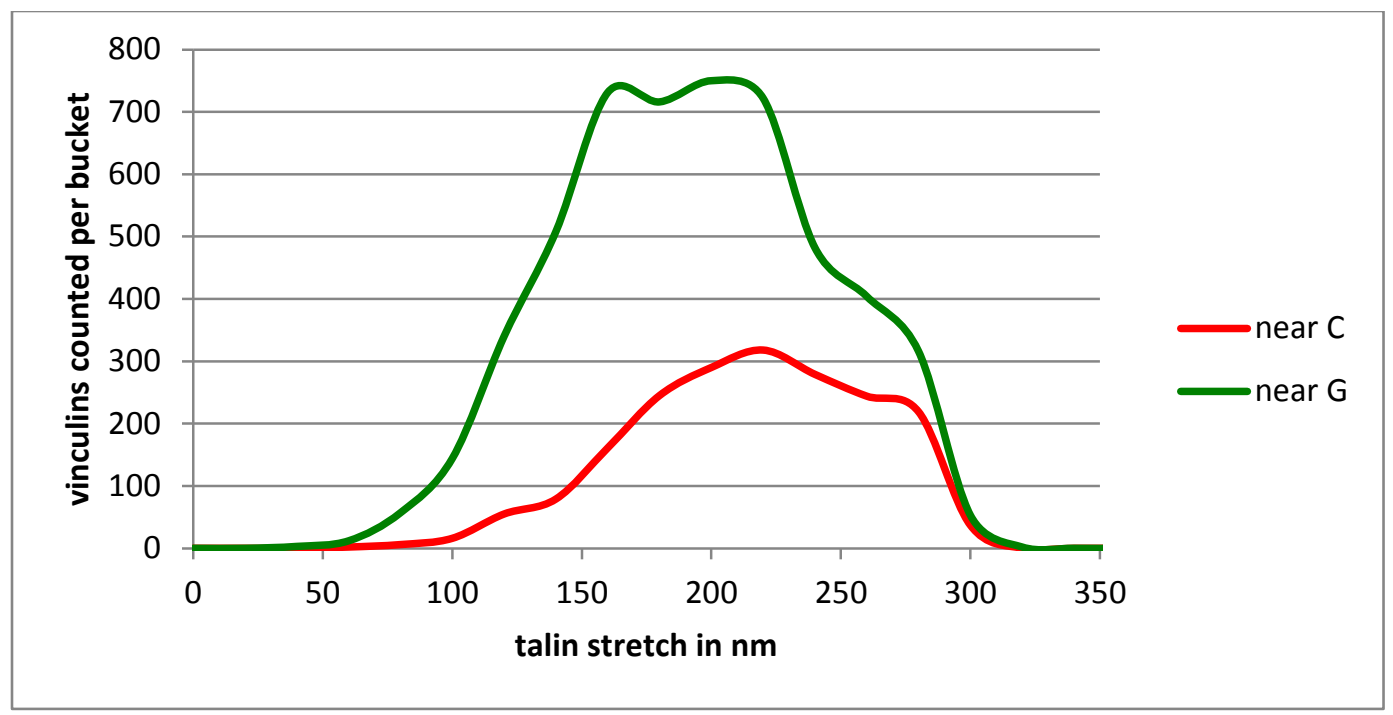

Supplementary Figure 7 Vinculin binding events depending on talin stretching length, separated into events near the $\mathbf{N}$ terminus and the $\mathrm{C}$ terminus respectively

A recruitment event was counted if the following criteria were met: it happened very proximal to the talin dimer and was either closer to the $\mathrm{C}$ terminus or the $\mathrm{N}$ terminus minus the resolution limit (about 20nm). Alternatively, it was assigned to one of the strands and was closer to the corresponding $\mathrm{N}$ or $\mathrm{C}$ terminus. Observations were also restricted to the areas of isolated adhesions. Of nearly $14 \mathrm{~K}$ events recorded in sparse adhesions with clear signals (via preselection of good recordings), some 5400 matched to $\mathrm{C}$ and 5200 matched to $\mathrm{N}$. We had to further delete the near-C recruitment events for which no ambiguity with either $\mathrm{N}$ terminus be excluded, lowering the useful near-C events to $2 \mathrm{~K}$. While the averages show a slight difference, the populations broadly overlap and exhibit a large variance. In harmony with the expectation that the $\mathrm{C}$ terminus binding sites are exposed at higher forces, the distribution shows a subtle preference for $\mathrm{C}$-recruitment at longer stretching distances. Because of the very large sample number, a non-uniform Welsh $t$-Test that produces the $t$ values of $t=-25.2$ for "the expectation value for $\mathrm{C}$ is greater or equal to $220 \mathrm{~nm}$ " and $\mathrm{t}=23.8$ for "the expectation value for $\mathrm{N}$ is smaller or equal to 200nm". The distributions are evidently non-normally distributed so that we used both a transformation, so we can use a Student's test (yielding ' $t$ 's of 4.48 and 4.23 respectively) and finally a Mann-Whitney rank test (binary $U$ test) with the counting variable $U$ now being neatly Gaussian and inverse Gaussian distributed: 
At stretches longer than $225 \mathrm{~nm} \mathrm{C}$ is the more likely terminal binding site is supported with $\mathrm{t}=37.4$ and the inverse for stretches $<200 \mathrm{~nm} \mathrm{~N}$ is the more likely terminal is supported with $\mathrm{t}=18.6$. These very high confidence intervals should not be regarded as hard evidence as the

processing chain and the automated sample selection introduce a substantial risk of bias. The number of events that contradict the trend is very large indeed.

\section{REFERENCE}

(1) Zhang, X.; Jiang, G.; Cai, Y.; Monkley, S. J.; Critchley, D. R.; Sheetz, M. P. Nat. Cell Biol. 2008, 10, (9), 1062-8.

(2) Margadant, F.; Chew, L. L.; Hu, X.; Yu, H.; Bate, N.; Zhang, X.; Sheetz, M. PLoS Biol. 2011, 9, (12), e1001223.

(3) Gallagher, S. S.; Sable, J. E.; Sheetz, M. P.; Cornish, V. W. ACS Chem. Biol. 2009, 4, (7), 547-56.

(4) Chen, Z.; Jing, C.; Gallagher, S. S.; Sheetz, M. P.; Cornish, V. W. J. Am. Chem. Soc. 2012, 134, (33), 13692-9.

(5) von Wichert, G.; Jiang, G.; Kostic, A.; De Vos, K.; Sap, J.; Sheetz, M. P. J. Cell Biol. 2003, 161, (1), 143-53. 